\title{
Effect of pioglitazone on outcome following curative treatment for hepatocellular carcinoma in patients with hepatitis $\mathrm{C}$ virus infection: A prospective study
}

\author{
SHUJI SUMIE ${ }^{1}$, TAKUMI KAWAGUCHI ${ }^{1}$, ATSUSHI KAWAGUCHI ${ }^{2}$, RYOKO KUROMATSU ${ }^{1}$, \\ MASAHITO NAKANO $^{1}$, MANABU SATANI ${ }^{1}$, SHINGO YAMADA ${ }^{1}$, SHUSUKE OKAMURA ${ }^{1}$, \\ YUKO YONEZAWA ${ }^{3}$, TATSUYUKI KAKUMA ${ }^{2}$, TAKUJI TORIMURA ${ }^{1}$ and MICHIO SATA ${ }^{1}$ \\ ${ }^{1}$ Division of Gastroenterology, Department of Medicine; ${ }^{2}$ Biostatistics Center; ${ }^{3}$ Graduate School of Medicine, \\ Kurume University School of Medicine, Kurume, Fukuoka 830-0011, Japan
}

Received July 24, 2014; Accepted September 1, 2014

DOI: $10.3892 / \mathrm{mco} .2014 .435$

\begin{abstract}
Pioglitazone is an insulin sensitizer used for the treatment of diabetes mellitus (DM). DM with insulin resistance is a risk factor for hepatocellular carcinoma (HCC) in patients with hepatitis $\mathrm{C}$ virus (HCV) infection. We aimed to investigate the effects of pioglitazone on $\mathrm{HCC}$ recurrence following treatment in $\mathrm{HCV}$-infected patients. Between 2009 and 2011, 85 HCV-infected HCC patients who underwent curative treatment were enrolled in this prospective study. Among 45 patients with type 2 DM, 27 were administered pioglitazone (pioglitazone group) following treatment. The remaining 58 patients were assigned to the control group. The primary outcome was recurrence-free survival. Changes in insulin resistance and serum adiponectin levels resulting from pioglitazone treatment were also assessed. In the whole analysis $(n=85)$, no significant difference in recurrence-free survival was observed between the pioglitazone and control groups. However, in a spline model analysis of DM patients, a decreased risk of HCC recurrence was associated with increased body weight in patients with a body mass index $(\mathrm{BMI}) \geq 23$; this association became significant at $\mathrm{BMI} \geq 24$ (hazard ratio $=0.17 ; 95 \%$ confidence interval: $0.03-0.95$ ). In addition, significantly decreased homeostasis model assessment for insulin resistance values $(\mathrm{P}=0.002)$ and significantly increased serum high-molecular-weight adiponectin levels $(\mathrm{P}<0.001)$ were observed following pioglitazone treatment. Although pioglitazone did not suppress $\mathrm{HCC}$ recurrence in the whole analysis, it inhibited HCC recurrence in overweight HCV-infected diabetic patients. Moreover, pioglitazone improved insulin resistance and adipocytokine levels. Thus,
\end{abstract}

Correspondence to: Dr Shuji Sumie, Division of Gastroenterology, Department of Medicine, Kurume University School of Medicine, 67 Asahi-machi Street, Kurume, Fukuoka 830-0011, Japan E-mail: sumie_shyuuji@kurume-u.ac.jp

Key words: pioglitazone, hepatocellular carcinoma, recurrence, obesity, diabetes mellitus, hepatitis $\mathrm{C}$ virus, insulin resistance pioglitazone may suppress HCC recurrence, which is associated with glucose and fat metabolism disorders.

\section{Introduction}

Hepatocellular carcinoma (HCC) is one of the most common malignancies worldwide. The incidence of HCC has increased in Eastern Asia and Africa over the last several decades and has also increased in the United States (1). In several countries, this trend is attributed to hepatitis $\mathrm{C}$ virus (HCV) infection and in Japan, $>70 \%$ of HCC cases are associated with chronic liver disease with $\mathrm{HCV}$ infection (2). Recent advances in imaging procedures and surveillance programs for high-risk patients have led to increased detection of early-stage HCC, resulting in an increase in the identification of patients in whom curative treatments, such as hepatic resection and radiofrequency ablation, may be feasible (3). However, as HCC frequently recurs even following curative treatment, prevention of recurrence is required to prolong the survival of $\mathrm{HCC}$ patients with $\mathrm{HCV}$ infection. For the prevention of HCC recurrence following curative treatment, adjuvant interferon therapy is known to be highly effective and is generally used in patients with $\mathrm{HCV}$ infection $(4,5)$. However, due to the high rate of non-response and severe adverse effects, interferon therapy is not universally used in HCV patients, particularly those who are older or who have a high viral load, genotype 1 and/or severe fibrosis $(6,7)$. Therefore, it is crucial to investigate other adjuvant therapies for the prevention of $\mathrm{HCC}$ recurrence following curative treatment in patients with $\mathrm{HCV}$ infection.

In chronic liver disease with HCV infection, the prevalence of type 2 diabetes mellitus (DM) has been reported to be higher compared to that associated with other chronic liver diseases, including hepatitis B virus infection (8). To explain this strong association, in addition to obesity, hepatic inflammation and fibrosis, Kawaguchi et al (9) and Shintani et al (10) suggested that HCV directly causes hepatic insulin resistance and subsequent hyperinsulinemia. Moreover, DM with insulin resistance was found to be a potential risk factor for the development of HCC, as well as for the recurrence of HCC in patients with HCV infection $(11,12)$. Thus, these findings led 
us to hypothesize that the treatment of type $2 \mathrm{DM}$ associated with insulin resistance significantly affects the development of $\mathrm{HCC}$ in patients with HCV infection.

Pioglitazone is a member of the thiazolidinedione family and is widely used for the treatment of type 2 DM. Pioglitazone reduces insulin resistance in the liver and peripheral tissues by stimulating the peroxisome proliferator-activated receptor (PPAR) $-\gamma$ and improves macrovascular outcomes (13). Previous studies reported that pioglitazone improves insulin resistance in patients with $\mathrm{HCV}$ infection treated with peginterferon and ribavirin $(14,15)$. In addition, pioglitazone itself was shown to exert anticarcinogenic activity through the inhibition of DNA synthesis and cell cycle progression in HCC cell lines and in an animal model of $\operatorname{HCC}(16,17)$. Moreover, pioglitazone was recently reported to suppress the onset of HCC in a hospital-based case-control study (18) and a population-based cohort study (19). Therefore, the aim of this study was to determine whether pioglitazone decreases the risk of HCC recurrence following curative treatment in patients with $\mathrm{HCV}$ infection. We also investigated the effect of pioglitazone on type $2 \mathrm{DM}$ due to insulin resistance.

\section{Patients and methods}

Patients. This clinical trial was conducted at the Kurume University School of Medicine. A total of 85 patients who met the inclusion criteria were enrolled between 2009 and 2011. The diagnosis of HCC was histologically confirmed by needle biopsy or based on the findings of typical radiological characteristics on dynamic computed tomography (CT) and magnetic resonance imaging (MRI). Pretreatment hepatic function was evaluated using the Child-Pugh scoring system. The inclusion criteria were i) HCV infection, ii) diagnosis of $\mathrm{HCC}$ with $\leq 3$ tumors, each $\leq 3 \mathrm{~cm}$, by imaging studies and iii) $\mathrm{HCC}$ treated with curative treatment (radiofrequency ablation or resection). The exclusion criteria were i) severe gastrointestinal stasis, ii) severe renal injury (creatinine $>2.0 \mathrm{mg} / \mathrm{dl}$ ), iii) severe esophageal and/or gastric varices, iv) HCC with macroscopic vascular invasion or extrahepatic metastasis, v) poorly differentiated HCC, vi) heart failure, vii) liver cirrhosis of Child-Pugh grade $\mathrm{C}$ and viii) type $1 \mathrm{DM}$.

The study protocol was approved by the Ethics Committee of Kurume University and conformed to the guidelines of the 1975 Declaration of Helsinki. Written informed consent was obtained from each subject prior to enrolment. This study has been registered in the University Hospital Medical Information Network (UMIN) Clinical Trials Registry under the registration number UMIN000007344.

Study design and pioglitazone treatment protocol. Patients who met the inclusion criteria were prospectively enrolled and were first evaluated for the presence of type 2 DM. Patients who did not have type $2 \mathrm{DM}$ were assigned to the control group (no treatment; $n=40$ ). For patients with type $2 \mathrm{DM}$, we additionally confirmed whether they wished to receive pioglitazone. Patients who declined administration of pioglitazone were assigned to the control group $(n=18)$ and patients who consented to receiving pioglitazone were assigned to the pioglitazone treatment group $(n=27)$. All the patients in the treatment group were administered pioglitazone at an initial dose of $30 \mathrm{mg} /$ day following curative treatment. Treatment discontinuation and dose reduction were based on toxicity. In cases with symptomatic heart failure, pioglitazone was permanently discontinued without dose reduction, regardless of the severity. In cases with facial and/or lower limb edema with functional impairment or symptomatic ascites, the patients were first treated with diuretics, such as spironolactone and furosemide. If symptomatic edema or ascites did not improve despite the administration of diuretics, pioglitazone was reduced to a dose of $15 \mathrm{mg}$ /day or interrupted until the symptoms disappeared. When weight gain $\geq 5 \%$ of baseline occurred, pioglitazone was reduced to a dose of $15 \mathrm{mg} /$ day. To evaluate the tolerability to pioglitazone, all the adverse events were recorded.

Diagnosis of type 2 DM and use of antidiabetic agents. Type 2 DM was classified according to the 2010 American Diabetes Association criteria (20). Patients with fasting plasma glucose $(\mathrm{FPG}) \geq 126 \mathrm{mg} / \mathrm{dl}, 2$-h plasma glucose $\geq 200 \mathrm{mg} / \mathrm{dl}$ during an oral glucose tolerance test, or a random plasma glucose $\geq 200 \mathrm{mg} / \mathrm{dl}$, were considered to have type 2 DM. Of the 85 patients included in this study, 45 were diagnosed with type $2 \mathrm{DM}$. Of the 45 patients with type $2 \mathrm{DM}$, no patients had been treated with pioglitazone prior to study enrolment and 24 patients were prescribed other antidiabetic agents, including biguanides $(n=2), \alpha$-glucosidase inhibitors $(n=11)$, sulfonylureas $(n=13)$ and insulin injections $(n=5)$.

Evaluation of type 2 DM control. In 27 of the 45 patients with type $2 \mathrm{DM}$, pioglitazone was administered following curative treatment. To evaluate the treatment effects of pioglitazone, body mass index (BMI), FPG, serum insulin level fasting immunoreactive insulin (FIRI), glycated hemoglobin (HbA1c) level and homeostasis model assessment for insulin resistance (HOMA-IR) values were measured at baseline and after 1-3 months. HOMA-IR values were calculated using the formula FPG (mg/dl) x FIRI (mU/l)/405. In addition, serum low-molecular-weight (LMW), middle-molecular-weight (MMW), high-molecular-weight (HMW) and total adiponectin levels were measured at baseline and after 1-3 months by enzyme-linked immunosorbent assay (ELISA) using the Human Adiponectin kit for Total and Multimers (Sekisui Medical Co., Ltd., Tokyo, Japan).

Follow-up and assessment of recurrence. Following curative treatment, each patient was closely followed up on a monthly basis. Serum biochemistry tests, $\alpha$-fetoprotein (AFP) levels and des- $\gamma$-carboxy prothrombin (DCP) levels were measured and ultrasonography was performed monthly. Contrast-enhanced dynamic CT was performed every 3 months until 6 months post-treatment and every 6 months thereafter. MRI was performed as a supplemental examination. Recurrence was diagnosed based on the combined findings of these assessments (characteristic appearances typical of HCC). The primary endpoint was recurrence-free survival, which was defined as the time interval between study entry and the first recurrence of HCC. Patients who survived without $\mathrm{HCC}$ recurrence were censored at the closing date of this study, which was December, 2011. The median duration of follow-up was 521 days (range, 100-961 days). 
Table I. Patient characteristics.

\begin{tabular}{|c|c|c|c|}
\hline Characteristics & Pioglitazone group $(\mathrm{n}=27)$ & Control group $(n=58)$ & P-value \\
\hline Age, years (range) & $72(53-84)$ & $70(50-86)$ & 0.832 \\
\hline Gender (male/female) & $17 / 10$ & $35 / 23$ & 0.818 \\
\hline BMI $\left(\mathrm{kg} / \mathrm{m}^{2}\right)$ & $22.9(20.1-30.7)$ & $23.0(15.2-29.3)$ & 0.401 \\
\hline $\operatorname{AST}(\mathrm{U} / \mathrm{l})$ & $53(23-137)$ & $52(17-118)$ & 0.257 \\
\hline $\operatorname{ALT}(\mathrm{U} / \mathrm{l})$ & $44(18-136)$ & $45(14-240)$ & 0.781 \\
\hline Platelet count $\left(\times 10^{9} / 1\right)$ & $107(46-185)$ & $111(37-281)$ & 0.431 \\
\hline Child-Pugh grade (A/B) & $22 / 5$ & $45 / 13$ & 0.682 \\
\hline Type 2 diabetes mellitus (present/absent) & $27 / 0$ & $18 / 40$ & $<0.001$ \\
\hline FPG (mg/dl) & $117(89-200)$ & $96(76-185)$ & \\
\hline FIRI $(\mu \mathrm{U} / \mathrm{ml})$ & $11.4(3.2-31.2)$ & $10.6(4.0-30.9)$ & \\
\hline HOMA-IR & $3.3(1.1-10.1)$ & $2.6(0.9-7.7)$ & \\
\hline HbA1c $(\%)$ & $6.1(4.7-8.0)$ & $5.3(4.5-8.6)$ & \\
\hline $\mathrm{AFP}(\mathrm{ng} / \mathrm{ml})$ & $14.8(2.3-282.1)$ & $13.4(2.4-710.4)$ & 0.393 \\
\hline $\mathrm{DCP}(\mathrm{mAU} / \mathrm{ml})$ & $21(11-8,220)$ & $25(8-486)$ & 0.688 \\
\hline Tumor size (mm) & $16(12-26)$ & $17(10-29)$ & 0.401 \\
\hline Tumor number (single/2-3) & $20 / 7$ & $37 / 21$ & 0.348 \\
\hline
\end{tabular}

Continuous variables are presented as median (range). BMI, body mass index; AST, aspartate aminotransferase; ALT, alanine aminotransferase; FPG, fasting plasma glucose; FIRI, fasting immunoreactive insulin; HOMA-IR, homeostasis model assessment for insulin resistance; HbA1c, glycated hemoglobin; AFP, $\alpha$-fetoprotein; DCP, des- $\gamma$-carboxy prothrombin.

Statistical analysis. Continuous variables are expressed as median (range) values. Comparisons between the two groups were performed using the Mann-Whitney U test for continuous variables and the Chi-square or Fisher's exact tests for discrete variables. Pioglitazone-induced metabolic changes were examined by the Wilcoxon's signed-rank test. Recurrence-free survival was measured by the Kaplan-Meier method and differences between subgroups were compared with log-rank tests. To investigate treatment efficacy in preventing $\mathrm{HCC}$ recurrence in the 45 patients with type $2 \mathrm{DM}$, we applied the Cox proportional hazards model, including not only the main terms BMI and group (pioglitazone or control) and their interaction term, but also knot terms for BMI that serve as inflection points. The Akaike information criterion (AIC) was used to evaluate these alternate models (21). The location of knots was determined objectively, with the minimum AIC among their prespecified candidates, which were whole-number BMI values from 15 to 30 . This model is referred to as a spline model, which we have previously described in detail $(22,23)$. Briefly, if the interaction term is selected, the hazard ratio (HR) between the pioglitazone and control groups has a functional form of BMI as the output of this model. All the P-values were two-tailed and $\mathrm{P}<0.05$ was considered to indicate a statistically significant difference. Statistical analysis was performed using SPSS software, version 20 (IBM Corp., Somers, NY, USA) and the R package version 3.0.1 (http://www.r-project.org/index.html).

\section{Results}

Patient characteristics. Patient characteristics are shown in Table I. The baseline characteristics of the two groups, including age, gender, BMI, aspartate aminotransferase level, alanine aminotransferase level, platelet count and Child-Pugh grade, were comparable, with no significant differences between groups. In addition, no significant differences in tumor characteristics, including AFP level, DCP level, tumor size, or tumor number, were observed. All the patients in the pioglitazone group and $18(32.0 \%)$ patients in the control group had type $2 \mathrm{DM}(\mathrm{P}<0.001)$.

\section{Effect of pioglitazone on prevention of recurrence}

Whole analysis. The recurrence-free survival curves of the 85 patients (whole analysis) are shown in Fig. 1A. The recurrence-free survival rates at 1 and 2 years were 84.6 and $44.2 \%$ respectively, in the pioglitazone group and 71.9 and $59.1 \%$ respectively, in the control group; none of these differences were statistically significant $(\mathrm{P}=0.9089)$.

Subanalysis of patients with type $2 \mathrm{DM}$

Log-rank test. The 45 patients with type $2 \mathrm{DM}$ were evaluated in a subanalysis. The recurrence-free survival curves of these patients are shown in Fig. 1B. The recurrence-free survival rates at 1 and 2 years were 84.6 and $44.2 \%$, respectively, in the pioglitazone group and 61.1 and $36.5 \%$, respectively, in the control group; none of these differences were statistically significant $(\mathrm{P}=0.3735)$.

Spline model analysis. The efficacy of pioglitazone in preventing HCC recurrence was evaluated by a spline model. Fig. 2 shows the association between BMI and HRs with 95\% confidence intervals (CIs) for HCC recurrence in the 45 patients with type $2 \mathrm{DM}$. In the Cox proportional hazards model, $\mathrm{BMI}=23$ was selected as the knot location (i.e., interaction term with group) and indicated a significant dependence of the HR between the pioglitazone and control groups for $\mathrm{BMI} \geq 23$. A significantly decreased risk of HCC recurrence 

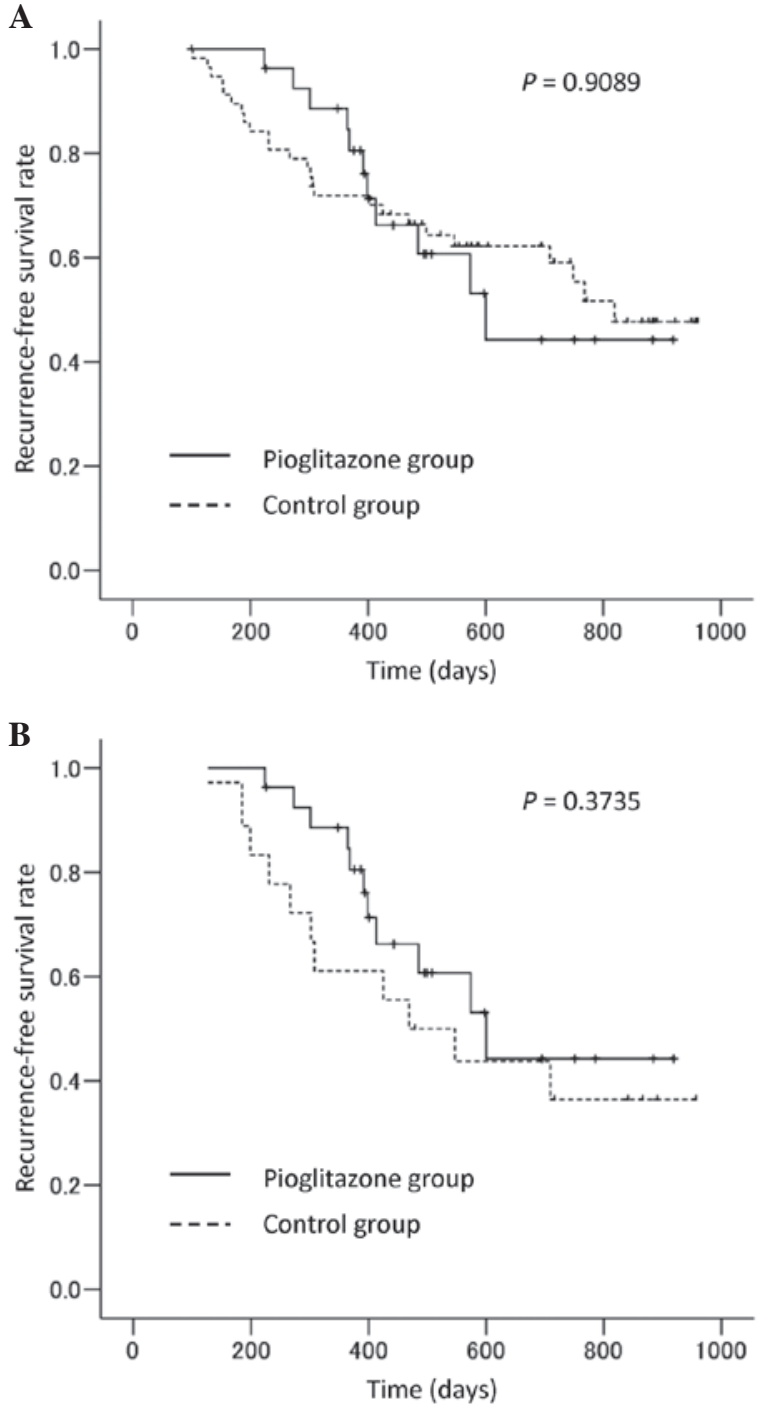

Figure 1. Recurrence-free survival curves of hepatocellular carcinoma patients with hepatitis $C$ virus infection who underwent curative treatment (A) Whole analysis of all 85 patients. (B) Subanalysis of 45 patients with type 2 diabetes mellitus.

was observed in the pioglitazone group for patients with a $\mathrm{BMI} \geq 24$ (HR=0.17; 95\% CI: 0.03-0.95).

Effect of pioglitazone on glucose metabolism and adiponectin levels. The changes in the metabolic variables in the 27 patients treated with pioglitazone are listed in Table II. BMI was significantly increased following pioglitazone treatment. By contrast, a significant improvement was observed in the glycemic variables, including FPG, FIRI, HOMA-IR and HbA1c. In addition, serum total, LMW, MMW and HMW adiponectin levels were significantly increased following pioglitazone treatment.

Adverse events. Of the 27 patients treated with pioglitazone, $18(66.7 \%)$ experienced $\geq 1$ adverse event. The most common adverse events were edema and subsequent weight gain, which occurred in $14(51.8 \%)$ and $8(29.6 \%)$ patients, respectively. These events were largely controlled by dose reduction of pioglitazone and diuretic treatment; however, 6 patients discontinued pioglitazone due to uncontrollable edema which

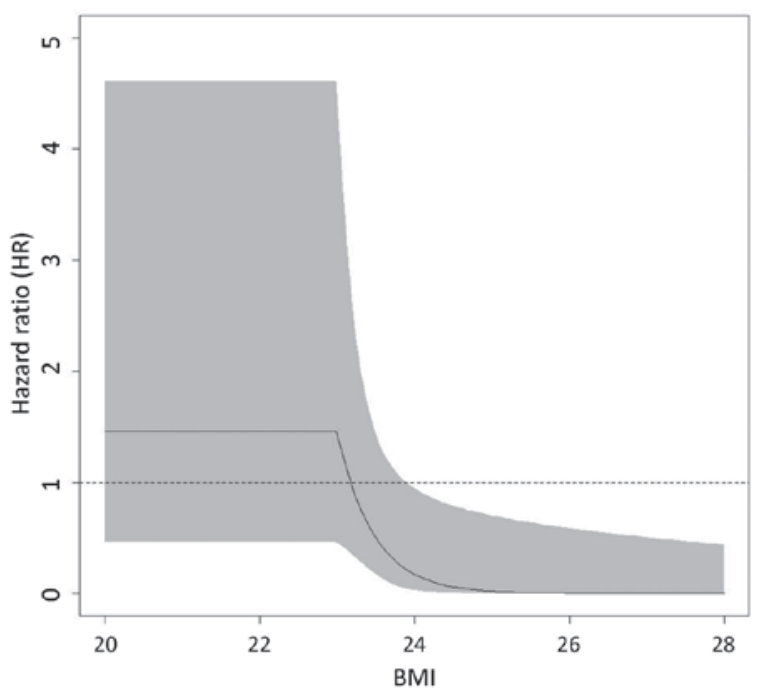

Figure 2. Association between body mass index (BMI) and hazard ratios (HRs) with $95 \%$ confidence intervals (CIs) for hepatocellular carcinoma (HCC) recurrence in the 45 patients with type 2 diabetes mellitus. A solid line and the gray area showed HRs and 95\% CIs, respectively. A significantly decreased risk of $\mathrm{HCC}$ recurrence in the pioglitazone group is observed for patients with a $\mathrm{BMI} \geq 24$.

inerfere with the activities of daily living. The median serum albumin levels in patients with and without edema were 3.4 and $3.9 \mathrm{mg} / \mathrm{dl}$, respectively $(\mathrm{P}<0.016)$. Moreover, the serum albumin level in all patients with edema was $<4.0 \mathrm{mg} / \mathrm{dl}$. Other mild adverse events included increased appetite and ascites in $3(11.1 \%)$ and $2(7.4 \%)$ patients, respectively. Two patients developed hypoglycemia, possibly caused by combination of insulin injections; this was controlled by reducing the insulin injections. Severe adverse events included acute heart failure in 1 patient, who discontinued pioglitazone $\sim 6$ months after treatment initiation. No patients developed liver dysfunction or gastrointestinal symptoms.

\section{Discussion}

In the whole analysis of this study, no significant differences in HCC recurrence-free survival rates were observed between the control and pioglitazone groups. However, a spline model analysis revealed that pioglitazone significantly decreased the risk of $\mathrm{HCC}$ recurrence in $\mathrm{HCV}$-infected diabetic patients with a $\mathrm{BMI} \geq 24$. Moreover, pioglitazone treatment decreased HOMA-IR values and increased serum multimeric adiponectin levels in HCV-infected diabetic patients. Thus, pioglitazone may inhibit HCC recurrence through the regulation of insulin resistance and adipocytokine levels in $\mathrm{HCV}$-infected diabetic patients who are overweight.

Pioglitazone, a PPAR $-\gamma$ agonist, inhibits DNA synthesis and cell cycle progression, resulting in anticarcinogenic activity in HCC cell lines and in an animal model of HCC $(16,17)$. Pioglitazone was recently reported to suppress the onset of HCC in a hospital-based case-control study (18) and a population-based cohort study (19). These findings suggest that pioglitazone may also exert inhibitory effects on HCC recurrence. However, the present results revealed no significant differences in $\mathrm{HCC}$ recurrence-free survival rates 
Table II. Changes in metabolic variables in 27 patients treated with pioglitazone.

\begin{tabular}{lccr}
\hline & \multicolumn{3}{c}{ Pioglitazone treatment } \\
\cline { 2 - 3 } Variables & Before & After & P-value \\
\hline Body weight $(\mathrm{kg})$ & $59.8(39.6-83.1)$ & $60.3(41.3-86.2)$ & 0.012 \\
FPG $(\mathrm{mg} / \mathrm{dl})$ & $117.5(89.0-200.0)$ & $110.0(80.0-159.0)$ & 0.007 \\
FIRI $(\mu \mathrm{U} / \mathrm{ml})$ & $11.3(3.2-31.2)$ & $9.3(1.8-27.7)$ & 0.006 \\
HOMA-IR & $3.3(1.1-10.0)$ & $2.6(0.6-7.1)$ & 0.002 \\
HbA1c $(\%)$ & $6.1(4.7-8.0)$ & $5.6(4.4-7.1)$ & 0.002 \\
Total adiponectin $(\mu \mathrm{g} / \mathrm{ml})$ & $8.8(1.5-49.3)$ & $26.8(3.1-60.7)$ & $<0.001$ \\
HMW adiponectin $(\mu \mathrm{g} / \mathrm{ml})$ & $4.3(0.2-32.4)$ & $17.3(0.9-39.1)$ & $<0.001$ \\
MMW adiponectin $(\mu \mathrm{g} / \mathrm{ml})$ & $1.6(0.4-9.1)$ & $4.1(0.9-12.6)$ & $<0.001$ \\
LMW adiponectin $(\mu \mathrm{g} / \mathrm{ml})$ & $3.7(0.9-7.8)$ & $5.6(1.4-13.0)$ & $<0.001$
\end{tabular}

Continuous variables are presented as median (range). FPG, fasting plasma glucose; FIRI, fasting immunoreactive insulin; HOMA-IR, homeostasis model assessment for insulin resistance; HbA1c, glycated hemoglobin; HMW, high-molecular-weight; MMW, middle-molecular-weight; LMW, low-molecular-weight.

between the control and pioglitazone groups in the whole analysis. Although the reason for the discrepancy between previous studies and the present findings remains unclear, the proportions of patients with HCV infection may be responsible for the differences between these results. All the patients included in our study were HCV-positive, whereas the majority of patients in the previous studies were not infected with $\mathrm{HCV}$. HCC frequently recurs due to various factors, including hepatic inflammation, hepatic fibrosis, HCV proteins, aging and therapeutic procedures in patients with HCV infection (24). Thus, pioglitazone may not have been sufficiently effective to universally suppress HCC recurrence in the present study.

We also performed a subanalysis of patients with type 2 DM. A log-rank test revealed no significant difference in HCC recurrence-free survival rates between the control and pioglitazone groups. However, the use of a Cox proportional hazards model, referred to as a spline model $(22,23)$, revealed that the HR decreased exponentially in association with increased body weight starting at $\mathrm{BMI}=23$ and this association became statistically significant at $\mathrm{BMI} \geq 24$. These findings revealed that being overweight is a key factor for pioglitazone-induced suppression of HCC recurrence. The overweight state causes insulin resistance and subsequent hyperinsulinemia (25). Since insulin is a growth-promoting hormone for HCC (26), we hypothesize that pioglitazone suppresses $\mathrm{HCC}$ recurrence through improvement of insulin resistance. In support of this hypothesis, we also demonstrated that pioglitazone significantly decreased HOMA-IR values, as well as serum insulin levels. An increased HOMA-IR value is a known risk factor for HCC recurrence (12), as is the use of antidiabetic agents that cause hyperinsulinemia, such as exogenous insulin and second-generation sulfonylurea agents (27), which further supports our hypothesis. Another mechanism underlying the development of HCC in overweight patients may be that overweight individuals exhibit increased expression of the pro-inflammatory cytokines tumor necrosis factor- $\alpha$ and interleukin-6 in adipose tissue, thereby causing hepatic inflammation and subsequent development of HCC (28).
These cytokines have also been demonstrated to be positively associated with the development of HCC in patients with $\mathrm{HCV}$ infection (29). Consequently, pioglitazone treatment may also suppress HCC recurrence through the downregulation of these cytokines in adipose tissue.

In this study, we also demonstrated that pioglitazone significantly increased multimeric adiponectin levels in $\mathrm{HCV}$-infected diabetic patients. Adiponectin is an adipocytokine that is inversely correlated with the amount of visceral fat accumulation, another important risk factor for HCC (30). Adiponectin not only increases insulin sensitivity, but was also shown to increase the levels of the tumor suppressor tuberous sclerosis, resulting in a significant reduction of liver tumorigenesis in an animal HCC model (31). We previously demonstrated that low serum adiponectin levels are associated with the development of HCC (32). Taken together, these findings suggest that the pioglitazone-induced upregulation of adiponectin may have contributed to the suppression of hepatocarcinogenesis observed in the present study. Moreover, lower adiponectin levels were recently reported to be associated with the malignant potential of HCC and poor prognosis in HCC patients $(31,33)$. Further studies should focus on the effects of pioglitazone on the overall survival of HCC patients.

The most common adverse events in this study were edema and subsequent weight gain. In particular, severe lower limb edema may compromise the quality of life of the patients. Thiazolidinedione-induced edema has been shown to occur through enhanced sodium-coupled bicarbonate absorption from the renal proximal tubules via PPAR- $\gamma$-dependent non-genomic signaling (34). In this study, patients with low serum albumin levels frequently experienced edema. Thus, physicians must be on the lookout for the development of pioglitazone-induced edema in patients with advanced liver disease. Although no patients in this study developed severe liver dysfunction, troglitazone, a first-generation thiazolidinedione, is known to cause severe liver failure (35). Troglitazone-induced changes in the hepatic molecular profiles differ from those caused by pioglitazone (36). However, thiazolidinedione-induced liver 
injury may occur through an idiosyncratic mechanism (37). In fact, fatal liver failure associated with pioglitazone has been previously reported (38). Therefore, we should also be aware of pioglitazone-induced liver injury in chronic liver disease with HCV infection.

In conclusion, the results of the present study demonstrated that pioglitazone did not suppress HCC recurrence in all patients. However, a spline model analysis revealed that pioglitazone significantly decreased the risk of HCC recurrence in $\mathrm{HCV}$-infected diabetic patients with a BMI $\geq 24$. Moreover, pioglitazone decreased HOMA-IR values and increased serum multimeric adiponectin levels in $\mathrm{HCV}$-infected diabetic patients. Thus, although pioglitazone does not appear to suppress HCC recurrence universally, it may exert an inhibitory effect on $\mathrm{HCC}$ recurrence in overweight $\mathrm{HCV}$-infected diabetic patients, possibly through the regulation of insulin resistance and adipocytokine levels.

\section{References}

1. El-Serag HB and Rudolph KL: Hepatocellular carcinoma: epidemiology and molecular carcinogenesis. Gastroenterology 132 2557-2576, 2007.

2. Kiyosawa K, Umemura $\mathrm{T}$, Ichijo $\mathrm{T}$, et al: Hepatocellular carcinoma: recent trends in Japan. Gastroenterology 127 (Suppl 1): S17-S26, 2004.

3. Noda I, Kitamoto M, Nakahara H, et al: Regular surveillance by imaging for early detection and better prognosis of hepatocellular carcinoma in patients infected with hepatitis $\mathrm{C}$ virus J Gastroenterol 45: 105-112, 2010.

4. Shiratori Y, Shiina S, Teratani T, et al: Interferon therapy after tumor ablation improves prognosis in patients with hepatocellular carcinoma associated with hepatitis $C$ virus. Ann Intern Med 138: 299-306, 2003

5. Mazzaferro V, Romito R, Schiavo M, et al: Prevention of hepatocellular carcinoma recurrence with alpha-interferon after liver resection in HCV cirrhosis. Hepatology 44: 1543-1554, 2006.

6. Iwasaki Y, Ikeda H, Araki Y, et al: Limitation of combination therapy of interferon and ribavirin for older patients with chronic hepatitis C. Hepatology 43: 54-63, 2006.

7. Fernandez-Rodriguez CM, Alonso S, Martinez SM, et al: Peginterferon plus ribavirin and sustained virological response in HCV-related cirrhosis: outcomes and factors predicting response. Am J Gastroenterol 105: 2164-2173, 2010.

8. Mason AL, Lau JY, Hoang N, et al: Association of diabetes mellitus and chronic hepatitis $C$ virus infection. Hepatology 29 : 328-333, 1999.

9. Kawaguchi T, Yoshida T, Harada M, et al: Hepatitis C virus down-regulates insulin receptor substrates 1 and 2 through up-regulation of suppressor of cytokine signaling 3. Am J Pathol 165: 1499-1508, 2004.

10. Shintani Y, Fujie H, Miyoshi $\mathrm{H}$, et al: Hepatitis $\mathrm{C}$ virus infection and diabetes: direct involvement of the virus in the development of insulin resistance. Gastroenterology 126: 840-848, 2004.

11. Chen CL, Yang HI, Yang WS, et al: Metabolic factors and risk of hepatocellular carcinoma by chronic hepatitis B/C infection: a follow-up study in Taiwan. Gastroenterology 135: 111-121, 2008.

12. Imai $\mathrm{K}$, Takai $\mathrm{K}$, Nishigaki $\mathrm{Y}$, et al: Insulin resistance raises the risk for recurrence of stage I hepatocellular carcinoma after curative radiofrequency ablation in hepatitis $\mathrm{C}$ virus-positive patients: a prospective, case series study. Hepatol Res 40: 376-382, 2010.

13. Miyazaki Y, Mahankali A, Matsuda M, et al: Improved glycemic control and enhanced insulin sensitivity in type 2 diabetic subjects treated with pioglitazone. Diabetes Care 24: 710-719, 2001.

14. Khattab M,Emad M, Abdelaleem A, et al: Pioglitazone improves virological response to peginterferon alpha-2b/ribavirin combination therapy in hepatitis $\mathrm{C}$ genotype 4 patients with insulin resistance. Liver Int 30: 447-454, 2010.

15. Harrison SA, Hamzeh FM, Han J, Pandya PK, Sheikh MY and Vierling JM: Chronic hepatitis $\mathrm{C}$ genotype 1 patients with insulin resistance treated with pioglitazone and peginterferon alpha-2a plus ribavirin. Hepatology 56: 464-473, 2012.
16. Rumi MA, Sato H, Ishihara S, et al: Peroxisome proliferator-activated receptor gamma ligand-induced growth inhibition of human hepatocellular carcinoma. Br J Cancer 84: 1640-1647, 2001.

17. Borbath I, Leclercq I, Moulin P, Sempoux C and Horsmans Y: The PPARgamma agonist pioglitazone inhibits early neoplastic occurrence in the rat liver. Eur J Cancer 43: 1755-1763, 2007.

18. Hassan MM, Curley SA, Li D, et al: Association of diabetes duration and diabetes treatment with the risk of hepatocellular carcinoma. Cancer 116: 1938-1946, 2010.

19. Lai SW, Chen PC, Liao KF, Muo CH, Lin CC and Sung FC: Risk of hepatocellular carcinoma in diabetic patients and risk reduction associated with anti-diabetic therapy: a population-based cohort study. Am J Gastroenterol 107: 46-52, 2012.

20. American Diabetes Association: Diagnosis and classification of diabetes mellitus. Diabetes Care 33 (Suppl 1): S62-S69, 2010

21. Akaike H: A new look at the statistical model identification. IEEE Trans Automat Control 19: 716-723, 1974.

22. Kawaguchi A, Yonemoto K, Tanizaki Y, Kiyohara Y, Yanagawa T and Truong YK: Application of functional ANOVA models for hazard regression to the Hisayama data. Stat Med 27: 3515-3527, 2008.

23. Tonan T, Fujimoto K, Qayyum A, et al: Quantification of hepatic iron concentration in chronic viral hepatitis: usefulness of T2-weighted single-shot spin-echo echo-planar MR imaging. PLoS One 7: e33868, 2012.

24. Ishikawa T: Strategy for improving survival and reducing recurrence of HCV-related hepatocellular carcinoma. World J Gastroenterol 19: 6127-6130,2013.

25. Rizvi AA: Metabolic markers of insulin resistance in overweight persons. Ann Intern Med 141: 243-244, 2004.

26. Saito K, Inoue S, Saito T, et al: Augmentation effect of postprandial hyperinsulinaemia on growth of human hepatocellular carcinoma. Gut 51: 100-104, 2002.

27. Singh S, Singh PP, Singh AG, Murad MH and Sanchez W: Anti-diabetic medications and the risk of hepatocellular cancer: a systematic review and meta-analysis. Am J Gastroenterol 108: 881-892, 2013

28. Park EJ, Lee JH, Yu GY, et al: Dietary and genetic obesity promote liver inflammation and tumorigenesis by enhancing IL-6 and TNF expression. Cell 140: 197-208, 2010.

29. Nakagawa H, Maeda S, Yoshida H, et al: Serum IL-6 levels and the risk for hepatocarcinogenesis in chronic hepatitis $\mathrm{C}$ patients: an analysis based on gender differences. Int $\mathbf{J}$ Cancer 125: 2264-2269, 2009

30. Ohki T, Tateishi R, Shiina S, et al: Visceral fat accumulation is an independent risk factor for hepatocellular carcinoma recurrence after curative treatment in patients with suspected NASH. Gut 58: 839-844, 2009.

31. Saxena NK, Fu PP, Nagalingam A, et al: Adiponectin modulates $\mathrm{C}$-jun $\mathrm{N}$-terminal kinase and mammalian target of rapamycin and inhibits hepatocellular carcinoma. Gastroenterology 139: 1762-1773, 2010.

32. Fukushima N, Kuromatsu R, Arinaga-Hino T, et al: Adipocytokine involvement in hepatocellular carcinoma after sustained response to interferon for chronic hepatitis C. Hepatol Res 40: 911-922, 2010.

33. Sumie S, Kawaguchi T, Kuromatsu R, et al: Total and high molecular weight adiponectin and hepatocellular carcinoma with HCV infection. PLoS One 6: e26840, 2011.

34. Endo Y, Suzuki M, Yamada H, et al: Thiazolidinediones enhance sodium-coupled bicarbonate absorption from renal proximal tubules via PPARgamma-dependent nongenomic signaling. Cell Metab 13: 550-561, 2011.

35. Kohlroser J, Mathai J, Reichheld J, Banner BF and Bonkovsky HL: Hepatotoxicity due to troglitazone: report of two cases and review of adverse events reported to the United States Food and Drug Administration. Am J Gastroenterol 95: 272-276, 2000.

36. Guo L, Zhang L, Sun Y, et al: Differences in hepatotoxicity and gene expression profiles by anti-diabetic PPAR gamma agonists on rat primary hepatocytes and human HepG2 cells. Mol Divers 10: 349-360, 2006.

37. Kaplowitz N: Avoiding idiosyncratic DILI: two is better than one. Hepatology 58: 15-17, 2013.

38. Chase MP and Yarze JC: Pioglitazone-associated fulminant hepatic failure. Am J Gastroenterol 97: 502-503, 2002. 